\title{
Erratum
}

\section{Unusual metal abundances in a pair of damped Lyman alpha systems at $z \sim 2^{\star}$}

\author{
S. L. Ellison ${ }^{1}$ and S. Lopez ${ }^{2}$ \\ 1 European Southern Observatory, Casilla 19001, Santiago 19, Chile \\ e-mail: sellison@eso.org \\ 2 Departamento de Astronomía, Universidad de Chile, Casilla 36-D, Santiago, Chile \\ e-mail: slopez@das.uchile.cl
}

A\&A 380, 117-122 (2001), DOI: 10.1051/0004-6361:20011431

In Table 2 of Ellison \& Lopez (2001) the value of [Si/H] in DLA A should be -1.04 . Although the subsequent [Si/Fe] is typical of other DLAs, a low ratio of $\alpha / \mathrm{Fe}$ is still supported by [S/Fe], compared with other systems in the literature. The moderate [Si/Fe] ratio could therefore be due to a modest amount of dust depletion, as supported by $[\mathrm{Zn} / \mathrm{Cr}]=0.18$. If dust is present, this would further decrease the already low [S/Fe] in DLA A. Therefore, despite the revised value of [Si/Fe] in DLA A, these results are still supportive of low $\alpha / \mathrm{Fe}$ in this double DLA. 